\title{
Video-Based Illumination Estimation
}

\author{
Ning Wang ${ }^{1,2}$, Brian Funt ${ }^{2}$, Congyan Lang ${ }^{1}$, and De $\mathrm{Xu}^{1}$ \\ ${ }^{1}$ School of Computer Science and Infromation Technology, \\ Beijing Jiaotong University, Beijing, China \\ ${ }^{2}$ School of Computing Science, Simon Fraser University, \\ 8888 University Drive, Burnaby, B.C, Canada \\ wangauss@gmail.com, funt@sfu.ca
}

\begin{abstract}
One possible solution to estimating the illumination for color constancy and white balance in video sequences would be to apply one of the many existing illumination-estimation algorithms independently to each video frame. However, the frames in a video are generally highly correlated, so we propose a video-based illumination-estimation algorithm that takes advantage of the related information between adjacent frames. The main idea of the method is to cut the video clip into different 'scenes.' Assuming all the frames in one scene are under the same (or similar) illuminant, we combine the information from them to calculate the chromaticity of the scene illumination. The experimental results showed that the proposed method is effective and outperforms the original single-frame methods on which it is based.
\end{abstract}

Keywords: color constancy, illumination estimation, scene cutting.

\section{Introduction}

Images recorded by a camera depend on three factors: the physical content of the scene, the illumination incident on the scene, and the sensitivity functions of the camera. The ability of a vision system to remove the effect of the illumination and recognize colors of objects independent of the light source is called color constancy [1]. The difficult problem in obtaining color constancy is to correctly estimate the chromaticity of the scene illumination. Once the scene illumination is determined, it can be used to transform the image so that it appears to be taken under a 'canonical', often white, light source. However, it is an ill-posed problem to determine the illumination chromaticity from the digital counts of an image. So only if additional assumptions are made, can the problem be solved. One such assumption is the Grayworld assumption, namely, that the average reflectance in the scene is achromatic. Under the Grayworld assumption, the illumination color is computed as the average RGB value of the image. The MaxRGB method makes a different assumption, namely, that the maximum digital count from each of the color channels taken separately represents the illumination color. Recent tests of this assumption indicate that it holds quite well [2]. A generalization of these two methods, called Shades of Gray [3], combines them using the Minkowski norm function. Other approaches based on more complex statistics of the image chromaticity distributions 
include the neural network approach [4], color by correlation [5], and the Bayesian approach [6]. All these algorithms are based on the zero-order structure of images, which means using the statistical distributions of the original pixel values to estimate the light source color of the image. In contrast, Van de Weijer et al. [7] have introduced a method based on the Grayedge hypothesis that uses higher-order structures (image derivatives) as the basis for illumination estimation.

These algorithms, however, are all designed in the context of still images. Although any one of them could be used on video by applying it on every single frame, no use would be made of the information from nearby frames. Since a video clip contains a series of still images that are related in time and, generally, in scene content as well, we propose a video-based illumination-estimation algorithm that takes advantage of the related information between adjacent frames. The main idea of the method is to cut the video clip into different 'scenes,' and then assuming that all the frames in the scene were taken under the same (or similar) illuminant, we combine the information from them to calculate the chromaticity of the scene illumination. It is reasonable to expect that combining the information across frames might improve performance since it is well known that the accuracy of most illumination-estimation algorithms improves as the number of distinctly colored surfaces increases [8]. Tests described below show that the video-based method is better than the frame-based approaches on which it relies.

The paper is organized as follows. In Section 2, the color image formation process is reviewed. In Section 3, a video-based illumination estimation algorithm is proposed. In Sections 4 and 5, the experimental results and the conclusions are presented.

\section{Color Image Formation}

The image digital counts, $\mathbf{f}=(R, G, B)^{T}$, for a Lambertian surface are the integral products of the illumination spectra $e(\lambda)$, the surface reflectance spectra $s(\lambda, \mathbf{x})$ and the camera sensitivity functions $\mathbf{r}(\lambda)=(R(\lambda), G(\lambda), B(\lambda))$,

$$
\mathbf{f}(\mathbf{x})=\int_{\omega} e(\lambda) s(\lambda, \mathbf{x}) \mathbf{r}(\lambda) d \lambda
$$

where $\mathbf{x}$ is the spatial coordinate in the image, $\lambda$ is the wavelength, and $\omega$ is the visible spectrum range. The goal of an illumination-estimation algorithm is to determine the illumination chromaticity (i.e., $r=R /(R+G+B), g=G /(R+G+B)$, and $b=B /(R+G+B))$ from the image values. This, however, is an ill-posed problem that cannot be solved without further assumptions. Grayworld, MaxRGB, Shades of Gray and Grayedge each make somewhat different assumptions, but they are all designed to solve for the illumination using only a single image frame as input. We will refer to them as frame-based (FB) methods. In contrast, we are proposing to use multiple frames from a video stream as input, which will refer to it as a video-based (VB) method. 


\section{Proposed Method}

An overview of the proposed video-based illumination-estimation algorithm is shown in Fig.1. There are four main steps. First of all, the illumination is estimated for each frame individually using one of the standard FB algorithms. Then using a scenecutting method, the video frames are sorted into different 'scenes.' Under the assumption that all frames within a single scene are lit by the same (or similar) illuminant, the information from them is combined to produce an estimate of the illuminant for the scene as a whole. This estimate is then also assigned to the scene's individual frames. The details of each step are discussed below.

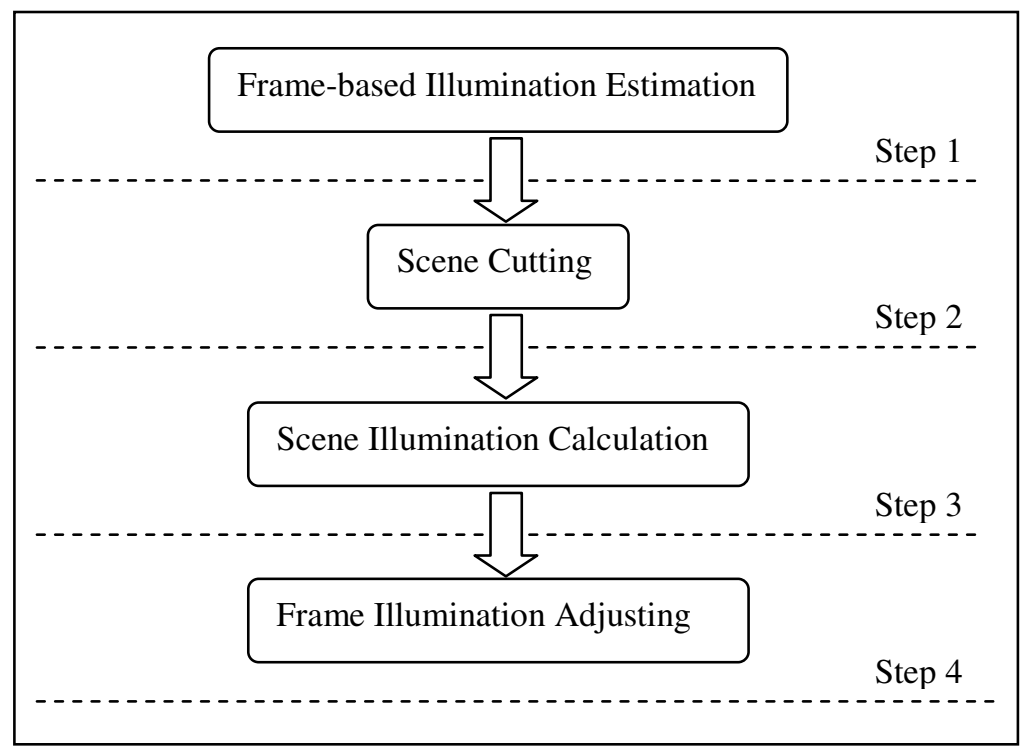

Fig. 1. Overview of video-based illumination estimation. See text for details.

\section{Step 1. Frame-Based Illumination Estimation}

For each frame, an FB algorithm is used to estimate its frame illumination color. Five commonly used FB methods-Grayworld (GW), MaxRGB, Shades of Gray (SoG), $1^{\text {st }}$ and $2^{\text {nd }}$ order Grayedge $(\mathrm{GE})$ - were tried. These particular methods are simple, unsupervised, fast, and sufficiently accurate to form a basis for the subsequent VB estimate. Of course, almost any other FB algorithm could be used instead.

The generalized edge-based framework proposed by van de Weijer et al. [7] is based on the Minkowski norm of the spatial image derivatives. The $\operatorname{rgb}$ color $\mathbf{e}^{n, p, \sigma}$ of the illumination is estimated as

$$
\left(\int_{\Omega}\left|\frac{\partial^{n} \mathbf{f}^{\sigma}(\mathbf{x})}{\partial \mathbf{x}^{n}}\right|^{p} d \mathbf{x}\right)^{1 / p}=k \mathbf{e}^{n, p, \sigma},
$$


where $n=1$ or 2 indicates the $1^{\text {st }}$ or $2^{\text {nd }}$ order of the spatial derivative of the image; $p \in[1, \infty)$ is the Minkowski norm; $\sigma$ is the standard deviation of a Gaussian filter; $k \in[0,1]$ is a scaling constant. An FB algorithm based on this equation assumes that the $p^{\text {th }}$ Minkowski norm of the derivative of the reflectance in a scene is achromatic. The five FB methods mentioned above can be viewed as special cases of this equation: $\mathbf{e}^{0,1,0}$ is Grayworld, $\mathbf{e}^{0, \infty, 0}$ is MaxRGB, $\mathbf{e}^{0, p, 0}$ is Shades of Gray, $\mathbf{e}^{1, p, \sigma}$ and $\mathbf{e}^{2, p, \sigma}$ are the $1^{\text {st }}$ and $2^{\text {nd }}$ order Grayedge algorithms.

Implementation details related to the FB algorithms are as follows. MaxRGB is the new version proposed by Funt et al. [2], which removes clipped pixels and applies a median filter to the image before computing the maxima of the color channels. For Shades of Gray and Grayedge, the choice of parameters is based on the results reported by van de Weijer et al. [7]. For every frame, the frame illumination color $\mathbf{F e}$ is computed using equation (2).

\section{Step 2. Scene Cutting}

The VB algorithm must determine which frames are under the same (or similar) illuminant as accurately as possible, or in other words, to determine the point at which the scene illumination changes. Ideally, the scene cutting would be based on the true illumination chromaticity, but then, of course, the illumination-estimation problem would have already been solved. Alternatively, the less-than-perfect FB estimate of the illumination in each frame is used to determine when the illumination has changed significantly. Change is measured in terms of the angular difference between the illumination chromaticity estimated for the current frame and the overall scene illumination estimated from the preceding frames. The angular difference $\theta$ computed as

$$
\theta(t)=\cos ^{-1}\left(\mathbf{F} \mathbf{e}_{t} \cdot \mathbf{S e} \mathbf{e}_{s}\right),
$$

where $\mathbf{F} \mathbf{e}_{t}$ is the FB estimated illumination chromaticity of frame $t$ and $\mathbf{S e}_{s}$ is the scene illumination chromaticity calculated from past frames that are contained in scene $s$. Given an angular threshold $\theta_{\text {Thres }}$, if $\theta(t)>\theta_{\text {Thres }}$, then $t-1$ is the end frame of the scene $s$ and $t$ is the first frame of next scene $s+1$. This will be called Angular Difference (AD) cutting.

Clearly, this method of scene cutting will fail when the FB estimate is unreliable. As an alternative, we also considered scene cutting based on measuring the change in scene content from frame to frame. The assumption is that sequential frames with similar content are likely lit by the same illuminant. This is implemented in terms of color histograms [10] with large changes in the histogram between frames used as an indication that the scene has changed. The histograms were defined in RGB color space of size $8 \times 8 \times 8$. The difference $\delta$ between color histograms is defined in terms of the Bhattacharyya [11] coefficient (a measure of their similarity) as:

$$
\delta(t)=1-\sum_{x \in X} \sqrt{h_{t}(x) h_{t+1}(x)},
$$


where $h_{t}(x)$ is the color histogram of frame $t$, and $X$ is the set of histogram bins. Given a threshold $\delta_{\text {Thres }}$, if $\delta(t)>\delta_{\text {Thres }}$, then $t$ is the last frame of the scene. This method is called color Histogram Difference (HD) cutting.

Of course, HD-based scene cutting also may fail at times so a third alternative is to combine the $\mathrm{AD}$ and HD methods in what will be termed Combined Difference (CD) cutting in the tables below. $\mathrm{CD}$ cuts a scene wherever either $\mathrm{AD}$ or $\mathrm{HD}$ or both would cut it.

\section{Step3. Scene Illumination Calculation}

Given a scene cut from a complete video along with the FB illumination estimate for each of its frames, the next step is the calculation of the overall scene illumination from the individual frame illuminations. This is done simply using either the mean or median of the frame estimates. The scene illumination for scene $s, \mathbf{S e}_{s}$, is calculated therefore either as

$$
\mathbf{S e}_{s}=\operatorname{mean}\left(\left\{\mathbf{F e}_{t}\right\}_{s}\right) /\left|\operatorname{mean}\left(\left\{\mathbf{F e}_{t}\right\}_{s}\right)\right|,
$$

or

$$
\mathbf{S e}_{s}=\operatorname{median}\left(\left\{\mathbf{F e}_{t}\right\}_{s}\right) /\left|\operatorname{median}\left(\left\{\mathbf{F} \mathbf{e}_{t}\right\}_{s}\right)\right|,
$$

where $\{\mathbf{F e}\}$ is the set of the FB illumination estimates for all frames in the scene, $s$ the index of the scene and $t$ is the index of the frame.

\section{Step 4. Frame Illumination Adjusting}

The last step of the VB algorithm is to replace the illumination estimate for each of the scene's frames with the newly calculated scene illumination:

$$
\mathbf{F e}_{t}^{\prime}=\mathbf{S e}_{s},
$$

where $\mathbf{S e}_{s}$ is the scene illumination of scene $s$ and $\mathbf{F e}_{t}^{\prime}$ is the adjusted frame illumination of frame $t$. Based on $\mathbf{F e}^{\prime}$, all the frames in the video clip could then be color balanced for the canonical illuminant using the von Kries coefficient rule.

\section{Testing the Video-Based Illumination-Estimation Method}

The video-based scene illumination method described above is tested on the gray-ball dataset presented by Ciurea and Funt [9]. This set contains 11,346 images extracted from about two hours digital video recorded with a Sony VX-2000 digital video camera. For each image, the ground-truth illumination is measured in terms of the RGB values of the pixels on a small gray ball attached in front of the camera (see Fig. 2 for example). The original images are 360x240 but are cropped to 240x240 in order to eliminate the gray ball. The dataset was originally presented as a test set for FB illumination-estimation methods, and as such the frames are at least $1 / 3$ second apart. Fortunately, the ordering of frames in the dataset corresponds to their original ordering in the video. Hence, sequences of the still frames represent a 
3-frame-per-second movie. In addition to having the ground-truth illumination available for every frame, this dataset has the advantage that it has been widely used in testing FB algorithms, thereby making comparison to these algorithms straightforward. The FB algorithms we compared here include the five methods mentioned in Section 3, Grayworld (GW), MaxRGB, Shades of Gray (SoG), and $1^{\text {st }}$ and $2^{\text {nd }}$ order Grayedge (GE).

For a single frame, the angular error between the ground-truth illumination chromaticity $\mathbf{e}_{a}=\left(r_{a}, g_{a}, b_{a}\right)$ and the estimated illumination chromaticity $\mathbf{e}_{t}=\left(r_{t}, g_{t}, b_{t}\right)$ is computed as

$$
\text { error }=\cos ^{-1}\left(\mathbf{e}_{a} \cdot \mathbf{e}_{t}\right) \text {. }
$$

Note that chromaticities are necessarily unit vectors. For the dataset as a whole, the median, mean, RMS and maximum angular errors computed across all frames is reported as well.

For the VB algorithm, there are three choices of cutting method (AD, HD, or CD) in Step 2, and two choices of combination method (mean versus median) in Step 3. In order to determine the best combination of choices, we first compare the use of mean versus median in Section 4.1, and then incorporate the better one with each of the three scene-cutting methods in Section 4.2. Finally, the performance of the VB algorithm using the optimal set of choices is compared to that of the FB algorithms in Section 4.3.
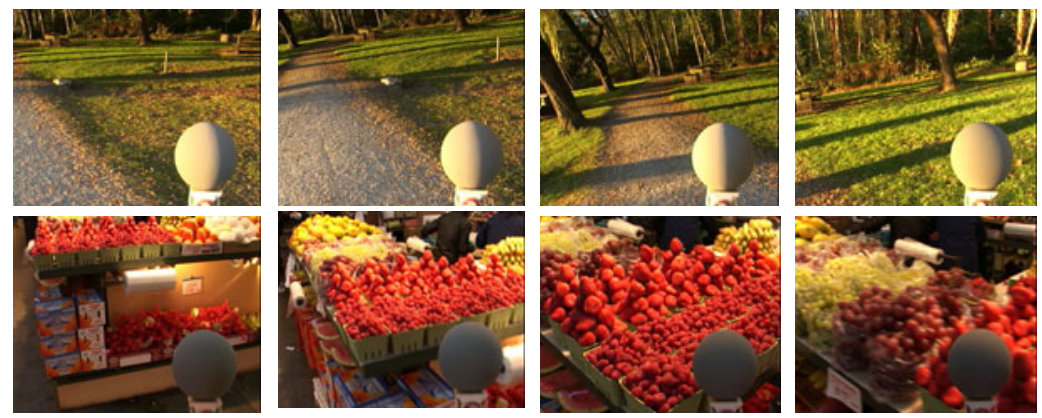

Fig. 2. Sample 3-frame-per-second image sequences from the 11,346 set [9]

\subsection{Comparison of Methods for Combining FB Estimates}

To test whether the using the mean or the median is better in terms of combining the estimates across a scene, and also to determine what the best possible result would be given a perfect scene cutting algorithm, we used the ground-truth illumination provided by the gray ball as the basis for scene cutting. For ground-truth illumination cutting, we computed the ground-truth illumination angular differences $\phi$ between neighboring frames,

$$
\phi(i)=\cos ^{-1}\left(\mathbf{T} \mathbf{e}_{i} \cdot \mathbf{T} \mathbf{e}_{i+1}\right),
$$


where Te is the ground-truth illumination and $i$ is the frame index. As with the other cutting methods, given an angular threshold $\phi_{\text {Thres }}$, if $\phi(i)>\phi_{\text {Thres }}$, then $i-1$ is the end frame of the scene $s$ and $i$ is the first frame of next scene $s+1$. The ground-truth illumination angular differences for all pairs of neighboring frames are shown in Fig. 3 (left), and their histogram is shown in Fig. 3 (right).
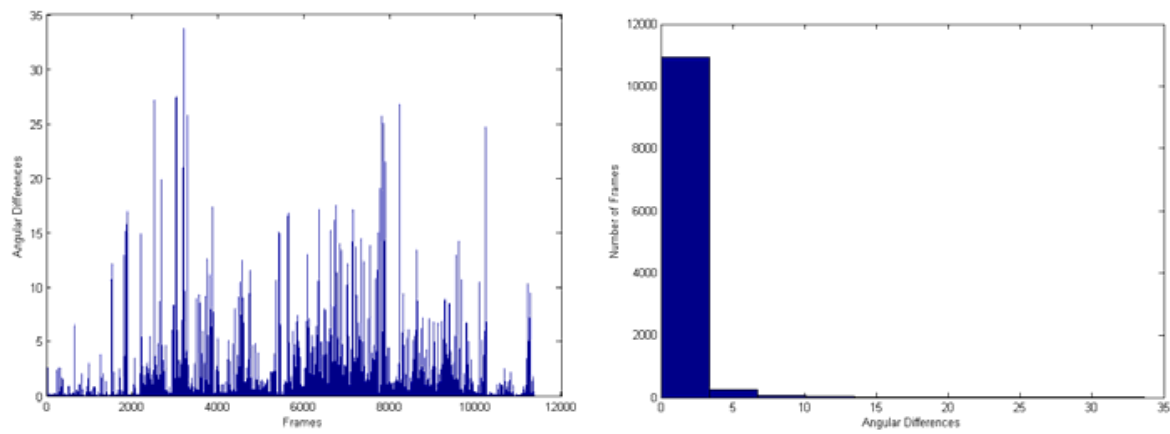

Fig. 3. (Left) Angular differences of ground-truth illumination between all pairs of neighboring frames. (Right) Corresponding histogram of the angular differences.

From Fig. 3 we can see that most of the angular differences are in the range [0, 5], and therefore select $\phi_{\text {Thres }}=5$ as the threshold. With this value, the 11,346 dataset is divided into about 380 scenes, which is close to what manual cutting produces. The VB algorithm's performance on the 11,346 set is listed in Table 1 as a function of the different FB methods with the FB results combined using either the mean or median. From this table, it is clear that generally the mean is slightly superior to the median as a combination strategy. Therefore, the mean is used in all subsequent tests.

Table 1. Comparison of mean versus median for combining FB estimates tested on the 11,346 dataset

\begin{tabular}{cccccc}
\hline \multirow{2}{*}{ FB Methods } & \multirow{2}{*}{$\begin{array}{c}\text { Scene Illumination } \\
\text { Calculation Methods }\end{array}$} & \multicolumn{4}{c}{ Angular Error } \\
\cline { 3 - 6 } & & Median & Mean & RMS & Max \\
\cline { 3 - 6 } & Mean & $\mathbf{4 . 5 7}$ & $\mathbf{5 . 9 6}$ & $\mathbf{7 . 4 6}$ & $\mathbf{3 3 . 2 6}$ \\
\multirow{2}{*}{ GW } & Median & 4.74 & 6.17 & 7.80 & 33.75 \\
\hline \multirow{2}{*}{ MaxRGB } & Mean & 5.06 & $\mathbf{6 . 4 9}$ & $\mathbf{8 . 3 6}$ & $\mathbf{2 4 . 4 6}$ \\
& Median & $\mathbf{4 . 9 9}$ & 6.69 & 8.62 & 24.83 \\
\hline \multirow{2}{*}{ SoG } & Mean & 4.10 & $\mathbf{5 . 0 6}$ & $\mathbf{6 . 1 9}$ & $\mathbf{2 9 . 6 6}$ \\
& Median & $\mathbf{3 . 9 9}$ & 5.13 & 6.23 & 29.91 \\
\hline \multirow{2}{*}{1 st GE } & Mean & $\mathbf{3 . 7 6}$ & $\mathbf{4 . 9 0}$ & $\mathbf{6 . 0 9}$ & $\mathbf{2 7 . 2 7}$ \\
& Median & 3.88 & 4.94 & 6.12 & 29.53 \\
\hline \multirow{2}{*}{2 2nd GE } & Mean & $\mathbf{3 . 8 6}$ & $\mathbf{5 . 0 5}$ & $\mathbf{6 . 2 2}$ & $\mathbf{2 6 . 5 7}$ \\
& Median & 3.97 & 5.08 & 6.22 & 26.70 \\
\hline
\end{tabular}




\subsection{Scene Cutting Methods Comparison}

As there are 15 images taken places in the 11346 set, we divided them into two nonoverlap subsets, one is for training and the other is for testing. In our experiments, we have 5618 images in the training set and 5728 images in the testing set. The training set is used to find out the optimal threshold values and the testing set is used to test.

The three scene cutting methods (AD, HD, CD) described above were tested in terms of the effect each has on the average VB angular error measure. In order to get the best value for the threshold for $\mathrm{AD}$, we tested the $\mathrm{VB}$ algorithm with $\theta_{\text {Thres }}$ ranging from 0 to 40 . The results are shown in Fig. 4 from which an appropriate threshold value for $\mathrm{AD}$ for each FB method can be determined. The thresholds chosen are: $\theta_{\text {Thres }}=20$ for Grayworld, $\theta_{\text {Thres }}=8$ for MaxRGB, $\theta_{\text {Thres }}=20$ for Shades of Gray, $\theta_{\text {Thres }}=12$ for $1^{\text {st }}$ and $2^{\text {nd }}$ order Grayedge.
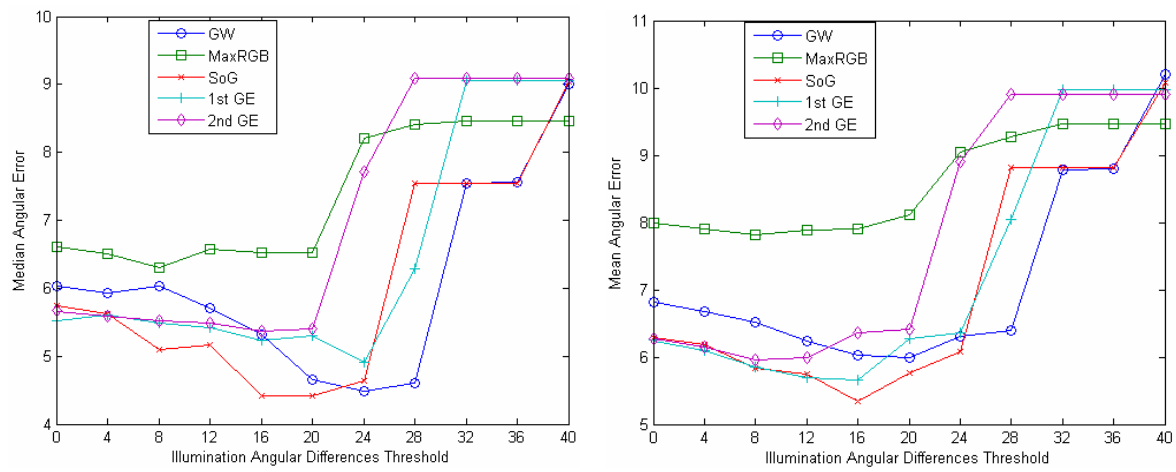

Fig. 4. Angular error as a function of threshold value used by $\mathrm{AD}$ for scene cutting tested on the training set. The threshold value is varied from 0 to 40 . Threshold $=0$ corresponds to every frame being a separate scene and therefore equivalent to FB. Left: median angular error. Right: mean angular error.
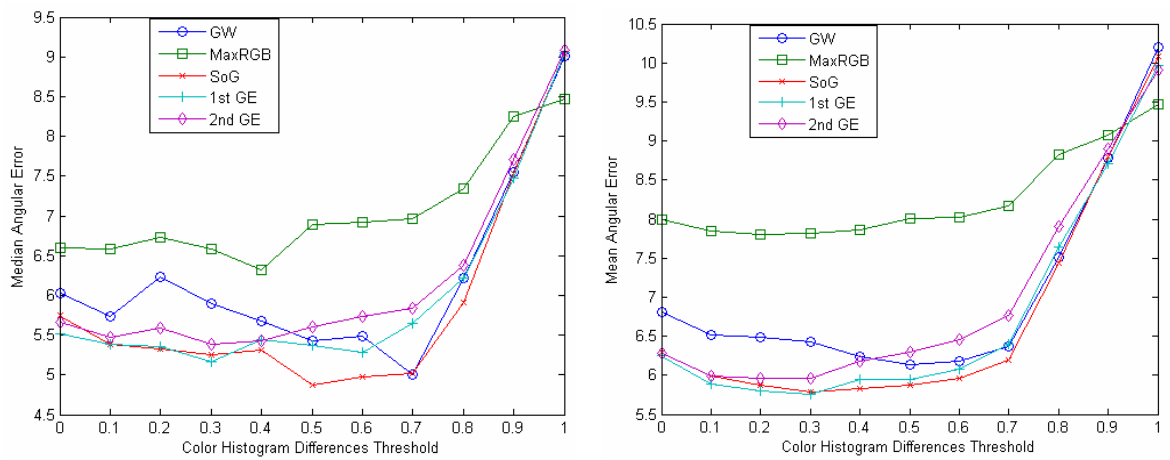

Fig. 5. The relationship between angular error and the threshold of HD on the training set. The threshold value is from 0 to 1 . Threshold $=1$ is equivalent to the corresponding FB method. Left: median angular error. Right: mean angular error. 
A similar test was conducted to determine the threshold values for optimal HD scene cutting. VB performance with $\delta_{\text {Thres }}$ ranging from 0 to 1 was evaluated, and the results are shown in Fig. 5. Based on these plots, the threshold values chosen were: $\delta_{\text {Thres }}=0.6$ for Grayworld, MaxRGB, Shades of Gray and $2^{\text {nd }}$ order Grayedge, and $\delta_{\text {Thres }}=0.5$ for $1^{\text {st }}$ order Grayedge.

Table 2 compares the performance of the VB algorithm as a function of both the scene-cutting and the FB methods being used on the testing set. Although the differences are not large, Table 2 shows that overall CD performs as well or better than either $\mathrm{AD}$ or HD applied separately. In the following tests, CD will be used as the scene cutting method.

Table 2. Video-Based performance on the testing dataset as a function of the scene-cutting method (angular difference, histogram difference, combined difference) and the FB (Greyworld, MaxRGB, Shades of Gray, Greyedge) method used

\begin{tabular}{|c|c|c|c|c|c|}
\hline \multirow{2}{*}{$\begin{array}{c}\text { FB } \\
\text { Methods }\end{array}$} & \multirow{2}{*}{ Scene Cutting Methods } & \multicolumn{4}{|c|}{ Angular Error } \\
\hline & & Median & Mean & RMS & Max \\
\hline \multirow{3}{*}{ GW } & AD: $\theta_{\text {Thres }}=20$ & 5.30 & 6.78 & 8.67 & 36.21 \\
\hline & HD: $\delta_{\text {Thres }}=0.6$ & 5.33 & 7.06 & 9.01 & 35.15 \\
\hline & $\mathrm{CD}: \theta_{\text {Thres }}=20, \delta_{\text {Thres }}=0.6$ & 5.29 & 6.99 & 8.65 & 35.96 \\
\hline \multirow{3}{*}{ MaxRGB } & $\mathrm{AD}: \theta_{\text {Thres }}=8$ & 3.46 & 5.43 & 7.20 & 28.01 \\
\hline & $\mathrm{HD}: \delta_{\text {Thres }}=0.6$ & 3.66 & 6.00 & 7.76 & 22.62 \\
\hline & $\mathrm{CD}: \theta_{\text {Thres }}=8, \delta_{\text {Thres }}=0.6$ & 3.43 & 5.48 & 7.19 & 28.01 \\
\hline \multirow{3}{*}{ SoG } & $\mathrm{AD}: \theta_{\text {Thres }}=20$ & 4.89 & 5.85 & 7.08 & 27.64 \\
\hline & $\mathrm{HD}: \delta_{\text {Thres }}=0.6$ & 4.93 & 5.96 & 7.40 & 28.81 \\
\hline & $\mathrm{CD}: \theta_{\text {Thres }}=20, \delta_{\text {Thres }}=0.6$ & 4.76 & 5.66 & 6.83 & 28.81 \\
\hline \multirow{3}{*}{ 1st GE } & $\mathrm{AD}: \theta_{\text {Thres }}=12$ & 3.80 & 5.29 & 6.86 & 32.09 \\
\hline & HD: $\delta_{\text {Thres }}=0.5$ & 3.68 & 5.29 & 6.61 & 27.89 \\
\hline & $\mathrm{CD}: \theta_{\text {Thres }}=12, \delta_{\text {Thres }}=0.5$ & 3.66 & 5.23 & 6.55 & 32.09 \\
\hline \multirow{3}{*}{ 2nd GE } & $\mathrm{AD}: \theta_{\text {Thres }}=12$ & 3.61 & 4.99 & 6.47 & 26.39 \\
\hline & HD: $\delta_{\text {Thres }}=0.6$ & 3.74 & 5.52 & 7.03 & 26.43 \\
\hline & $\mathrm{CD}: \theta_{\text {Thres }}=12, \delta_{\text {Thres }}=0.6$ & 3.60 & 4.89 & 6.33 & 26.38 \\
\hline
\end{tabular}

\subsection{Comparing to FB Existing Algorithms}

Based on the tests in Sections 4.1 and 4.2, the VB algorithm should be based on combining the mean of the FB results across scenes cut using the CD (combine difference method using both inter-frame illumination and color histogram differences). The remaining question is the extent to which VB improves upon the FB result for each of the FB methods. Table 3 compares the results of VB employing each of the 5 FB algorithms. From this table, we can see that when using the VB 
algorithm for illumination-estimation, all the error measures-median, mean, RMS and maximum-decreased, in some cases by as much as $20 \%$. Table 3 shows that video-based illumination-estimation, which makes use of information from multiple frames within a scene, although simple, is quite effective.

Table 3. Comparison of the VB and FB methods on the 11,346 set

\begin{tabular}{cccccc}
\hline \multirow{2}{*}{ Methods } & & \multicolumn{4}{c}{ Angular Error } \\
\cline { 2 - 5 } & & Median & Mean & RMS & Max \\
\cline { 2 - 5 } & & 6.34 & 7.36 & 8.99 & 43.82 \\
\multirow{2}{*}{ GW } & FB & $5.08(-19.9 \%)$ & $6.24(-15.2 \%)$ & $7.78(-13.5 \%)$ & $34.33(-21.7 \%)$ \\
\hline \multirow{2}{*}{ MaxRGB } & FB & 5.30 & 6.85 & 8.78 & 28.38 \\
& VB & $4.86(-8.3 \%)$ & $6.60(-3.6 \%)$ & $8.62(-1.8 \%)$ & $28.01(-1.3 \%)$ \\
\hline \multirow{2}{*}{ SoG } & FB & 5.33 & 6.17 & 7.51 & 36.04 \\
& VB & $4.19(-21.4 \%)$ & $5.30(-14.1 \%)$ & $6.50(-13.4 \%)$ & $28.18(-21.8 \%)$ \\
\hline \multirow{2}{*}{1 st GE } & FB & 5.12 & 6.09 & 7.54 & 34.14 \\
& VB & $4.44(-13.3 \%)$ & $5.51(-9.5 \%)$ & $6.87(-8.9 \%)$ & $32.09(-6.0 \%)$ \\
\hline \multirow{2}{*}{2 nd GE } & FB & 5.11 & 5.98 & 7.33 & 32.15 \\
& VB & $4.11(-19.6 \%)$ & $5.38(-10.0 \%)$ & $6.71(-8.5 \%)$ & $26.78(-16.7 \%)$ \\
\hline
\end{tabular}

\section{Conclusion}

By utilizing the information available from a contiguous set of frames from a video sequence, video-based estimates of the chromaticity of the scene illumination improved by as much as $20 \%$ over estimates based on single frames in isolation. The video-based method simply averages the illumination estimates of the frames taken individually. However, it is crucial to be able to determine when in the video sequence the scene illumination scene has changed such that the subsequent frames should not be included in the average. Scene cutting based on measuring both change in the frame-based illumination estimates and change in scene content quantified in terms of color histograms proved effective.

\section{Acknowledgment}

This work is supported by National Nature Science Foundation of China (60803072) and (61033013), and the Natural Sciences and Engineering Research Council of Canada.

\section{References}

1. Forsyth, D.A.: A Novel Algorithm for Color Constancy. International Journal of Computer Vision 5(1), 5-36 (1990)

2. Funt, B., Shi, L.: The Rehabilitation of MaxRGB. In: Proc. IS\&T Eighteenth Color Imaging Conference, San Antonio (2010)

3. Finlayson, G.D., Trezzi, E.: Shades of gray and colour constancy. In: Color Imaging Conference, IS\&T - The Society for Imaging Science and Technology, pp. 37-41 (2004) 
4. Cardei, V.C., Funt, B., Barnard, K.: Estimating the scene illumination chromaticity by using a neural network. J. Opt. Soc. Am. A. 19(12), 2374-2386 (2002)

5. Finlayson, G.D., Hordley, S.D., Hubel, P.M.: Color by correlation: A simple, unifying framework for color constancy. IEEE Trans. Pattern Analysis and Machine Intelligence 23(11), 1209-1221 (2001)

6. Brainard, D.H., Longère, P., Delahunt, P.B., Freeman, W.T., Kraft, J.M., Xiao, B.: Bayesian model of human color constancy. Journal of Vision 6, 1267-1281 (2006)

7. van de Weijer, J., Gevers, T., Gijsenij, A.: Edge-based color constancy. IEEE Trans. Image Proc. 16(9), 2207-2214 (2007)

8. Barnard, K., Martin, L., Coath, A., Funt, B.: A comparison of computational color constancy algorithms - part ii: Experiments with image data. IEEE Trans. Image Proc. 11(9), 985-996 (2002)

9. Ciurea. F., Funt, B.: A Large Image Database for Color Constancy Research. In: Proc. Eleventh Color Imaging Conference, pp. 160-164. The Society for Imaging Science and Technology (2003)

10. Swain, M.J., Ballard, D.H.: Color indexing. Int. J. Comput. Vision, 11-32 (1991)

11. Djouadi, A., Snorrason, O., Garber, F.D.: The Quality of Training Sample Estimates of the Bhattacharyya Coefficient. IEEE Trans. Pattern Anal. Mach. Intell., 92-97 (1990) 